\title{
Hydraulic forces on rails for vertical lift, high head gates
}

\author{
D. G. Wardle and D. A. Crow
}

Mr M. J. Kenn (Fellow), formerly Imperial College, London

This Paper had been read with great interest because flow visualization studies for an unusual gate slot (rather similar to that of the Authors) were undertaken at Imperial College, London, on models with significantly differing linear scaics and in contrasting situations, both with and without cavitation. ${ }^{7}$ The latter studies were incidental to other associated investigations. However, the patterns of twodimensional flows showed remarkable similarities (Figs 9-11) and were remarkably similar to the Authors' sketched flow patterns in their Figs 1 and $7(b)$ for three-dimensional flows. It would be interesting to know the Reynolds numbers for the latter flows (based on slot width and mean velocities passing the slot). It would also be interesting to see photographs of the Authors' flow patterns.

31. The present flow patterns (Figs 9-11) were obtained, respectively, in: (i) a low-speed water tunnel, with a linear scale of $1: 5$, a mean flow velocity of $2.7 \mathrm{~m} / \mathrm{s}$ and with entrained air used to portray the waterflow pattern (Fig. 9); (ii) a small elevated cavitation tunnel, with a linear scale of $1: 52$, a mean flow velocity of $21.0 \mathrm{~m} / \mathrm{s}$ (at a pressure of 0.5 atmospheres (absolute)) and with cavitation used to portray the flow pattern (Fig. 10); (iii) an Ahlborn tank, with a linear scale of $1: 8$, a mean flow velocity of the order of $0.30 \mathrm{~m} / \mathrm{s}$ and with aluminium powder used to portray the free-surface water flow pattern (Fig. 11).

32. The similarity of the flow pattern (Fig. 9), obtained at relatively low velocity and without cavitation, with that (Fig. 10) obtained at high velocity and with cavitation, was of particular significance. Based on slot width, the Reynolds numbers for these flows were quite similar, at $5.2 \times 10^{5}$ and $3.9 \times 10^{5}$, respectively, but both were rather less than that of the full-sized structure, at $3.4 \times 10^{7}$. Also of significance, however, was the almost identical pattern of flow (Fig. 11) obtained in the Ahlborn tank, at the rather lower Reynolds number of about $0.4 \times 10^{5}$.

33. An additional Ahlborn-tank test, made without the protruding rail on the downstream face of the gate slot, gave rise to a much simpler pattern of flow, with far less eddying (Fig. 12). The sensitivity of the pattern of flow to the presence (Fig. 11), or not (Fig. 12), of a protruding guide rail was most striking, and demonstrated that small changes of boundary detail could give rise to profound (and dominant) changes of flow patterns.

Paper published: Proc. Instn Civ. Engrs, Part 2, 1985, 79, Mar., 73-85. 


\section{DISCUSSION}

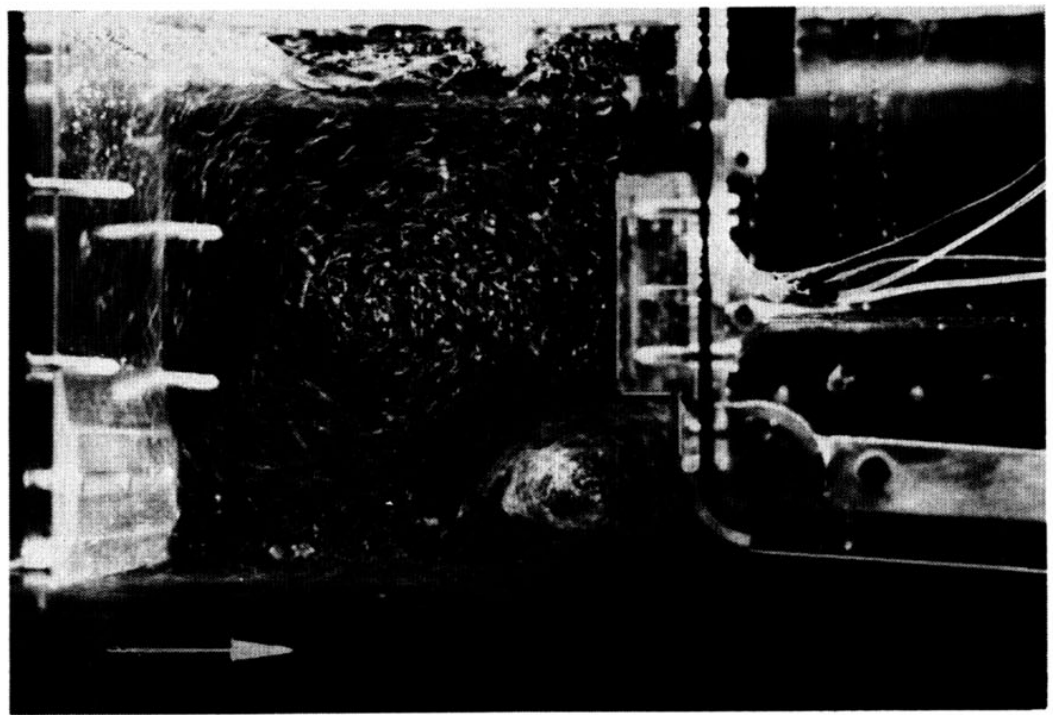

Fig. 9. Flow pattern (with entrained air) for $1: 5$ scale model of gate slot with protruding guide rail. Tested in a water tunnel at a mean flow velocity of $2.7 \mathrm{~m} / \mathrm{s} .\left(R_{e}=5.2 \times 10^{5}\right.$, based on slot width)

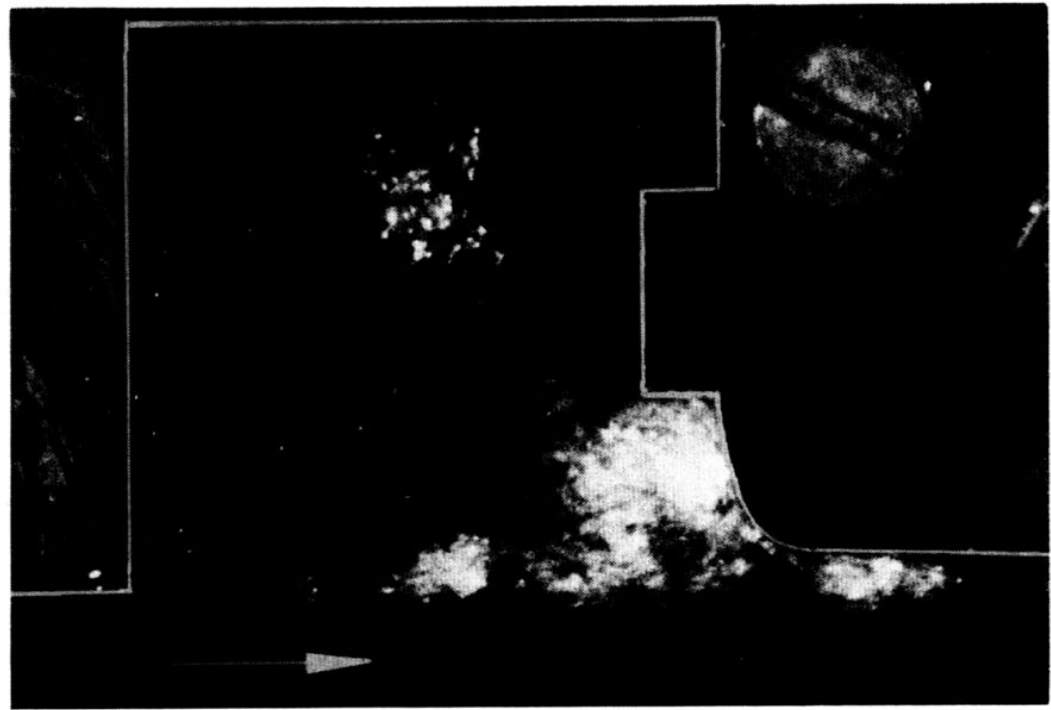

Fig. 10. Flow pattern (with cavitation) for $1: 52$ scale model of gate slot with protruding guide rail. Tested in an elevated cavitation tunnel at a mean flow velocity of $21.0 \mathrm{~m} / \mathrm{s}$ and a pressure of 0.5 atmospheres (absolute). $\left(R_{e}=3.9 \times 10^{5}\right.$, based on slot width) 


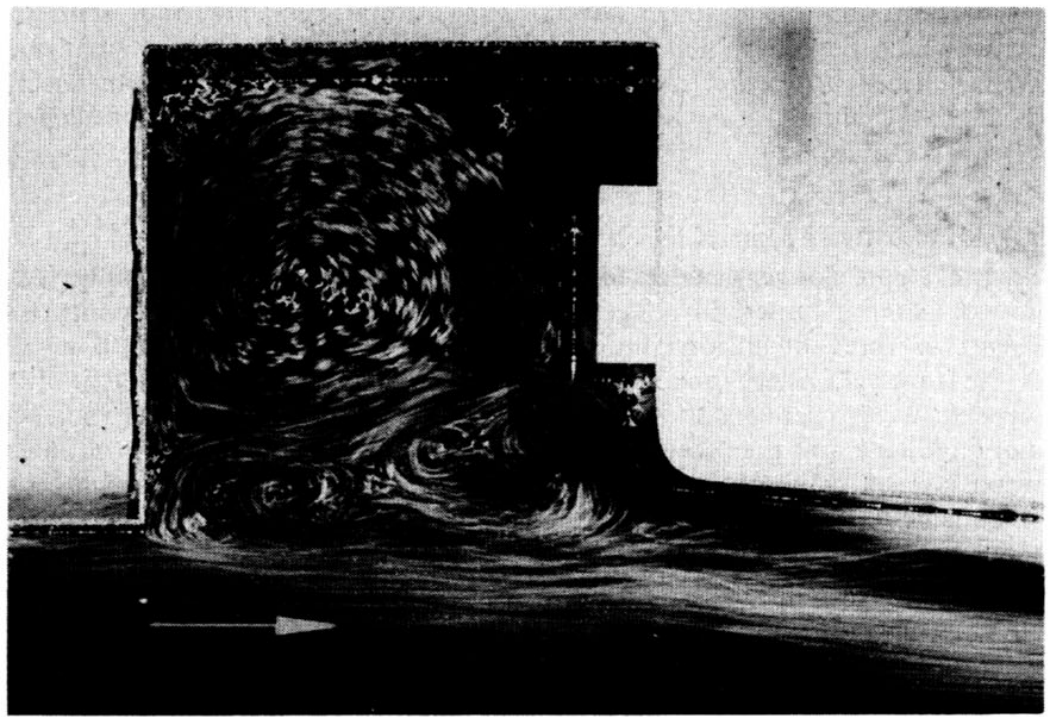

Fig. 11. Flow pattern (with aluminium powder on the free water surface) for $1: 8$ scale model of gate slot with protruding guide rail. Tested in an Ahlborn tank with a mean flow velocity of $0.3 \mathrm{~m} / \mathrm{s} .\left(R_{e}=0.4 \times 10^{5}\right.$, based on slot width $)$

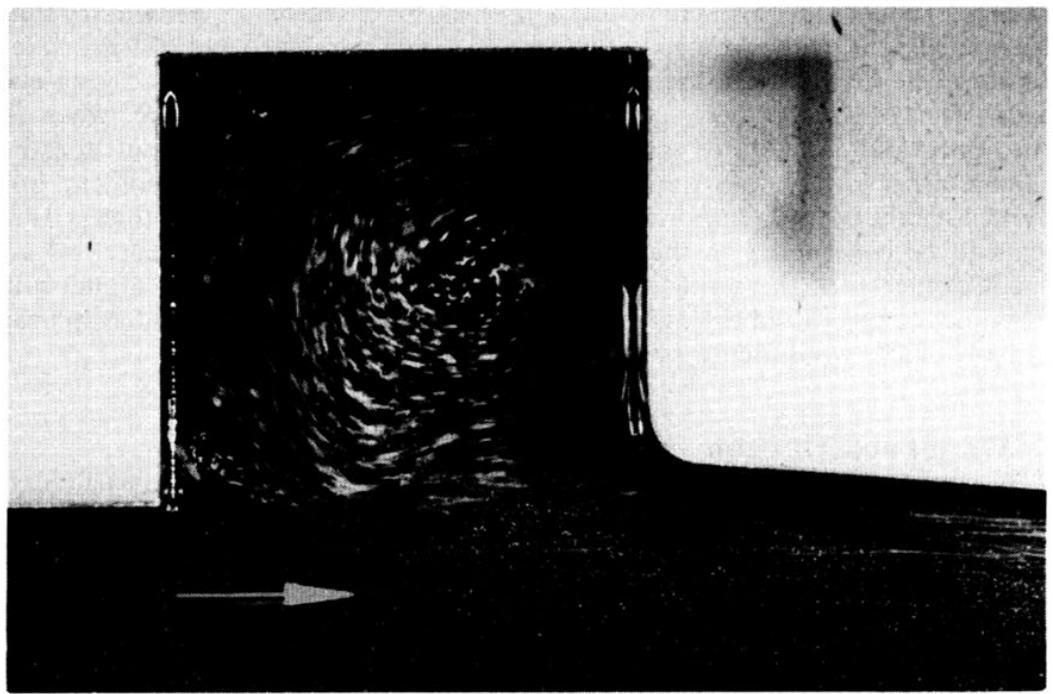

Fig. 12. Flow pattern (with aluminium powder on the free water surface) for $1: 8$ scale model of gate slot without protruding guide rail. Tested in an Ahlborn tank with a mean flow velocity of $0.3 \mathrm{~m} / \mathrm{s} .\left(R_{e}=0.4 \times 10^{5}\right.$, based on slot width $)$ 
34. These, and other, studies have also shown that significant and useful data can, with care, be determined from relatively simple tests and that a small-scale model, if tested in a water tunnel at full-scale heads and velocities can usefully indicate likely patterns of cavitation (and even cavitation erosion) for the elements of a large structure.

\section{Dr J. D. Hardwick, Imperial College, London}

Studies of a gate slot very similar to that treated by the Authors were conducted in a flow of water at Imperial College on a model scale of about $1: 5$. In contrast to the Authors' three-dimensional model, which reproduced the full height of the gate slot, the Imperial College model was two-dimensional, reproducing a height of slot equivalent to $3 b$ where $b$ is the width of the slot shown in Fig. 8 of the Paper. Figs 5 and 6 indicate that the pressure coefficient near the north-east corner of the rail approached $1 \cdot 0$, while the two-dimensional model showed a value which corresponds fairly closely with the measurements of Ball and Tinney, Robertson and Bennett, ${ }^{1,6}$ which were also made on two-dimensional models. Another marked difference was in the force tending to push the rail away from its seating. Fig. 6 shows that near the floor, the difference between the pressure coefficients for the east and west sides of the rail could be in the region of $(0 \cdot 9-0 \cdot 27)=0.63$. The corresponding difference for the Imperial College model was rather less. It would be interesting to know what difference the Authors observed on their rail when their model was made two-dimensional.

36. The Authors argue that the upward flow in the gate slot has an important influence on both the flow pattern and the forces on the rail. The writer subsequently constructed a simple but three-dimensional $1: 80$ scale model of a slot in a flow channel and confirmed that there was indeed a strong upward flow of water in the slot. The model also confirmed a prediction (based on an elementary theoretical treatment by the writer) that water will rise in the slot to a height equivalent to about $0 \cdot 13 \bar{V}^{2} / 2 g$ above the free surface of the mainstream near the vena contracta. The $\bar{V}^{2} / 2 g$ for these tests was equivalent to only about $20 b$, when the prototype value is likely to exceed $50 \mathrm{~b}$, and the theoretical treatment does not account either for the constriction of the slot by the gate or air entrainment, both of which might be more important for higher values of $\bar{V}^{2} / 2 g$. Nethertheless, there seems to be consistency in the Authors' measurements and the theoretical and practical checks of the writer. This suggests that the Authors' air model must indeed have reproduced the dynamics of the flow in the slot with greater accuracy than any of the two-dimensional models.

\section{Mr Wardle and Mr Crow}

We are grateful to $M r$ Kenn and $D r$ Hardwick for their contributions, particularly in view of the similar geometries of the gate slots which were tested.

38. As noted in $\$ \$ 19$ and 23 of our Paper, we investigated the flow patterns in the gate slots when flows were restricted to two dimensions as opposed to the more complex three-dimensional flow which had been observed in the full model. Most of these tests were made on a flow table (that is, Ahlborn tank) at Reynolds numbers, based on the slot width, of between 0.1 and $0.2 \times 10^{5}$, which is comparable with the condition shown by Mr Kenn in Fig. 11. In the absence of photographic records, we attempted to illustrate these contrasting flow patterns in our Figs $7(a)$ and $7(b)$. 
39. The similarities in flow patterns observed by $M r$ Kenn in his twodimensional flow tests is clear to see in his Figs 9 and 10, and to a lesser degree in Fig. 11, which shows a more complex pattern of vortices on the open side of the slot. In the latter case, which was a similar arrangement to our flow table tests, the differences may have been attributable to the sheared velocity profile from the floor of the tank.

40. Although $\mathrm{Mr}$ Kenn sees a remarkable similarity between his twodimensional flow patterns and our three-dimensional flow shown in Fig. 7(b), there is in fact a significant difference in that the three-dimensional flow pattern is characterized by the absence of any secondary vortices at the open side of the slot. The fact that there is a vertical component of flow up the slot means that at any given horizontal section, there can be a nett flow from the tunnel into the slot. This radically alters the flow pattern as the flow in the tunnel between the tunnel wall and the stagnation streamline, can flow steadily into the slot. In the case of two-dimensional flow, no nett flow into (or out of) the slot is possible, although considerable energy is fed into the slot by way of shear forces. It is our view that this accounts for the generally unstable nature of the two-dimensional flow patterns and the continual shifting, growth and decay of the several vortices formed.

41. In Fig. 12, Mr Kenn shows the altered flow pattern obtained when the protruding rail was removed and observes that small changes in boundary detail give rise to profound changes of flow pattern. While agreeing with this assertion, which had been previously noted by one of the present Authors, ${ }^{8}$ we believe that restricting the three-dimensional flow pattern to two dimensions constitutes a much more significant change in boundary 'detail', which so distorts the flow patterns as to invalidate most of the data obtained from two-dimensional tests.

42. Dr Hardwick's contribution to the discussion is of particular interest as he has confirmed, using a water model at a much smaller scale, that there is a strong upward flow in the slot. Furthermore, it is interesting that he has addressed the problem theoretically, and encouraging that he has obtained some experimental confirmation of his analysis.

\section{References}

7. KENN M. J. Flow visualization aids cavitation studies. IAHR/DVWK Symp. on Scale Effects in Modelling Hydraulic Structures, Esslingen, 1984, Sept., 1-6.

8. Coxon R. E., Angelin S. V. and Wardle D. G. Stoplog closure model prototype correlation. J. Hydraul. Div. Am. Soc. Civ. Engrs, 1973, 99, Feb., HY2, 301-317. 